\title{
Point vortex model for prediction of sound generated by a wing with flap interacting with a passing vortex
}

\author{
A. Manela ${ }^{a)}$ \\ Faculty of Aerospace Engineering, Technion - Israel Institute of Technology, Haifa 32000, Israel \\ L. Huang \\ Department of Mechanical Engineering, The University of Hong Kong, Pokfulam Road, Hong Kong
}

(Received 22 October 2012; revised 14 January 2013; accepted 24 January 2013)

\begin{abstract}
Acoustic signature of a rigid wing, equipped with a movable downstream flap and interacting with a line vortex, is studied in a two-dimensional low-Mach number flow. The flap is attached to the airfoil via a torsion spring, and the coupled fluid-structure interaction problem is analyzed using thin-airfoil methodology and application of the emended Brown and Michael equation. It is found that incident vortex passage above the airfoil excites flap motion at the system natural frequency, amplified above all other frequencies contained in the forcing vortex. Far-field radiation is analyzed using Powell-Howe analogy, yielding the leading order dipole-type signature of the system. It is shown that direct flap motion has a negligible effect on total sound radiation. The characteristic acoustic signature of the system is dominated by vortex sound, consisting of relatively strong leading and trailing edge interactions of the airfoil with the incident vortex, together with late-time wake sound resulting from induced flap motion. In comparison with the counterpart rigid (non-flapped) configuration, it is found that the flap may act as sound amplifier or absorber, depending on the value of flap-fluid natural frequency. The study complements existing analyses examining sound radiation in static- and detached-flap configurations.
\end{abstract}

(C) 2013 Acoustical Society of America. [http://dx.doi.org/10.1121/1.4792246]

PACS number(s): 43.28.Ra, 43.28.Py, 43.50.Nm [PBB]

Pages: $1934-1944$

\section{INTRODUCTION}

Airframe noise, and in particular sound generated by high-lift devices, is known to be a major cause for acoustic radiation, particularly during airplanes' approach for landing. ${ }^{1}$ Significant efforts have therefore been made to analyze the sound generated by such devices in various setups and flight conditions. ${ }^{2-6}$ Common to almost all of these works is a static configuration of a detached lift device, where a slot separates the device from the main body of the airfoil. The outcome acoustic field in such cases is significantly affected by vortex shedding and flow separation occurring at the gap. To reduce trailing edge sound radiation, several works have been focusing on developing related noise-control methodologies. ${ }^{7,8}$

In parallel with aeroacoustic studies of static aerodynamic configurations, analyses of vibroacoustic phenomena, coupling between thin-structure motions and ambient fluid flows, have recently attracted considerable attention owing to their relevance to various applications. Specific examples include, among others, effects of acoustic disturbances on aerodynamic performance of micro-air-vehicle wings, ${ }^{9}$ as well as insect flight sound, ${ }^{10-12}$ in which small thickness-tochord wing ratios are encountered. Motivated by these phenomena, theoretical investigations of the motion and acoustic signature of elastic structures have been carried out, both in the dynamical ${ }^{13-16}$ and acoustic ${ }^{17,18}$ contexts.

The key idea of the present contribution is to incorporate the vibroacoustic methodology developed in the above-

\footnotetext{
a) Author to whom correspondence should be addressed. Electronic mail: avshalom@aerodyne.technion.ac.il
}

mentioned studies to consider the effect of lift-device motion on its acoustic radiation. Unsteady flow conditions will be modeled in the form of incoming flow vorticity, and their nonlinear coupling with flap dynamics will be examined. Unlike detached-flap configurations studied previously, we consider an attached-flap setup, where the flap is hinged to an airfoil through an elastic torsion spring. This choice of a setup is motivated by recent investigations of continuous "mold-line link" flap configurations, ${ }^{19,20}$ which aim at reducing sound levels emitted by high lift devices in commercial air transports. Introduction of elastic degree of freedom for the flap is inevitable in these cases to enable efficient flap actuation. ${ }^{21}$ Noting this application, the present work may be relevant in two cases of interest: first, for the study of sound levels emitted by a connected flap setup in a regular-scale wing; and second, as a first step for examining the impact of trailing edge elasticity, common in small-scale flight, on wing acoustic radiation.

The model problem to be studied considers a small Mach number setup. For the calculation of the near-field flow, we therefore assume the flow to be incompressible to obtain a leading order description. Flow compressibility is taken into account for evaluating the far-field sound. Under these conditions, the system source region can be treated as acoustically compact, and the compact Green's function theory can be applied for the acoustic calculation. ${ }^{22,23}$ The theoretical scheme is expected to yield a reasonable approximation in cases where the mean-flow Mach number $M$ is small enough so that $M^{2} \ll 1$ (Ref. 22). These conditions also hold in the above-mentioned experimental studies of attached-flap configurations ${ }^{19,20}$ where $M \lessgtr 0.2$, and are prevalent in small-scale flight of micro-air-vehicles and insects. 
The paper outline is as follows: in Sec. II, the non-linear problem for near-field flow and flap dynamics is formulated, and in Sec. III the problem for the far-field acoustic pressure is presented. Both dynamical and acoustic problems are analyzed in Sec. IV, and respective numerical results are given in Sec. V. Concluding comments are given in Sec. VI. Technical details are relegated to the Appendix.

\section{FLAP DYNAMICS AND NEAR-FIELD FLOW}

Consider a two-dimensional airfoil of chord $2 a$, consisting of a stationary upstream part, aligned with the $x_{1}$-axis, and attached to a flap at $x_{1}=\bar{\eta} a$ (with $0<\bar{\eta}<1$, see Fig. 1). The flap is hinged to the airfoil through a torsion spring of constant $k_{\theta}$, and the system is subject to low-Mach highReynolds number flow of speed $U$ in the $x_{1}$-direction. An incident line vortex of strength $\Gamma$ is released into the flow at a given location at time $t=0$ and moves past the airfoil-flap system. We assume fluid vorticity to be concentrated at the incident vortex location and along a trailing edge wake, whereas the rest of the near flow-field is assumed irrotational and treated by means of potential-flow theory. In what follows we analyze the flap dynamics, governed by its time-dependent angular deflection $\theta(t)$ from the $x_{1}$-axis, together with the associated fluid motion. It is postulated, and later on verified, that $|\theta(t)| \ll 1$, so that linearized thin-airfoil theory can be applied to study the near-field flow, and release of leading edge vorticity can be neglected at the outset. In addition, it is assumed that the flap is fully attached to the airfoil with no slot separating between them. As mentioned in Sec. I, by this we intend to complement existing studies focusing on the sound radiated from detached and static flap configurations.

We formulate an initial value problem, where, at time $t=0$, the flap is aligned with the $x_{1}$-axis $(\theta=0)$ and has zero angular velocity $(\mathrm{d} \theta / \mathrm{d} t=0)$. At the initial time, the incident vortex $\Gamma$ is set into the flow at a prescribed location, and its motion is followed for $t>0$. We denote the instantaneous vortex position and velocity at time $t$ by $\mathbf{x}_{\Gamma}(t)$ and $\mathbf{v}_{\Gamma}(t)=\mathrm{d} \mathbf{x}_{\Gamma} / \mathrm{d} t$, respectively. To acquire finite flow velocity at the airfoil trailing edge, the unsteady Kutta condition needs to be applied. This necessitates release of vorticity from the flap end point into a trailing edge wake.

We model the evolution of trailing edge wake through the Brown and Michael equation, originally suggested for the evaluation of lift on delta wings. ${ }^{24,25}$ According to this model, the shedding of vorticity is discretized into a sequence of line vortices whose position and strength vary with time. At any given time, one vortex is being shed from the airfoil trailing edge, in the form of a thin connecting sheet of infinitesimal circulation

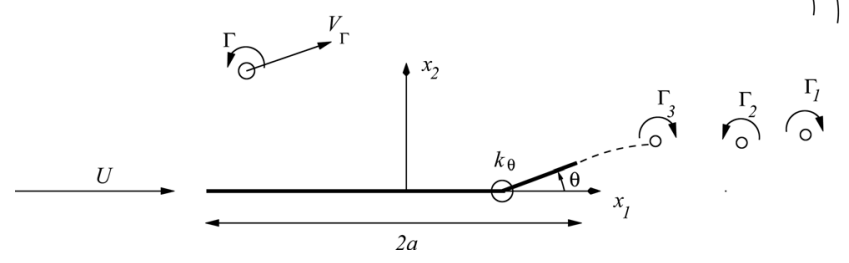

FIG. 1. Schematic of the fluid-airfoil system. ending in a concentrated core of finite circulation $\Gamma_{n}$. The core strength changes with time according with the Kutta condition, while its position $\mathbf{x}_{\Gamma n}$ is governed by the Brown and Michael formula. When the time derivative $\mathrm{d} \Gamma_{n} / \mathrm{d} t$ changes sign, the vortex is detached from the thin sheet and propagates as a "free" line vortex with "frozen" (fixed) circulation. Simultaneously, the shedding of another vortex, $\Gamma_{n+1}$, is initiated.

While the original Brown and Michael formula has been applied to model vortex shedding dynamics in various configurations, it has been shown to induce a spurious dipole, resulting from the time dependence of the circulation of the growing vortex. Several corrections have been proposed to overcome this problem (e.g., Refs. 26 and 27), among which Howe $^{28}$ has derived an emended form of the equation, suitable for aeroacoustic calculations of far-field radiation from two-dimensional airfoils at high Reynolds number flows. In what follows, we apply Howe's form of the Brown and Michael equation [see (9)] to model the shedding of trailing edge vortices. A similar approach has been followed by Guo, ${ }^{29}$ who studied the sound radiated from a stationary Joukowski airfoil using the Ffowcs Williams-Hawkings acoustic analogy. It should be noted that, strictly, Howe's emended equation is valid for vortex shedding from stationary structures only. We therefore consider our present use of Howe's theory as a leading order approximation, justified by the small amplitude of flap motion. An extension of Howe's formula to consider vortex shedding from non-stationary structures is outside the scope of the present contribution. ${ }^{30}$

Making use of thin-airfoil methodology, we represent the airfoil by distribution of vorticity $\gamma_{a}\left(x_{1}, t\right)\left(-a \leq x_{1} \leq a\right)$ per unit length. To specify $\gamma_{a}\left(x_{1}, t\right)$, the impermeability condition on the structure is imposed. In complex-variable notation, this condition is given by the integral equation

$$
\begin{aligned}
\frac{\partial h}{\partial t}+U \frac{\partial h}{\partial x_{1}}= & \operatorname{Im}\left\{\frac { i } { 2 \pi } \left[\int_{-a}^{a} \frac{\gamma_{a}(s, t) \mathrm{d} s}{x_{1}-s}+\frac{\Gamma}{x_{1}-z_{\Gamma}}\right.\right. \\
& \left.\left.+\sum_{k=1}^{n} \frac{\Gamma_{k}}{x_{1}-z_{\Gamma_{k}}}\right]\right\},
\end{aligned}
$$

where $z=x_{1}+\mathrm{i} x_{2}$ is a complex representation of a point in the plane of motion, and $z_{\Gamma}$ and $z_{\Gamma_{k}}$ are the locations of incident and $k$ th trailing edge vortices, respectively. The barred integral sign marks a principal value integral, and $h$ denotes the $x_{2}$-displacement of the airfoil,

$$
h\left(x_{1}, t\right)= \begin{cases}0, & -a \leq x_{1} \leq \bar{\eta} a \\ \left(x_{1}-\bar{\eta} a\right) \theta(t), & \bar{\eta} a<x_{1} \leq a .\end{cases}
$$

Thus, the left-hand and right-hand sides in Eq. (1) equate the normal components of airfoil and fluid-induced velocities at the wing surface, respectively.

The flap angular motion is governed by a balance between the rate of change of flap angular momentum and moments applied by the torsion spring and fluid-loading force around the fixed hinge location. Explicitly,

$$
I_{f} \frac{\mathrm{d}^{2} \theta}{\mathrm{d} t^{2}}=-k_{\theta} \theta+\int_{\bar{\eta} a}^{a} \Delta p\left(x_{1}, t\right)\left(x_{1}-\bar{\eta} a\right) \mathrm{d} x_{1} .
$$


In Eq. (3), $I_{f}$ is the flap moment of inertia per unit span around the hinge point, and $\Delta p=p_{-}-p_{+}$is the pressure jump across the airfoil, with $p_{-}$and $p_{+}$denoting fluid pressures at the airfoil lower and upper surfaces, respectively. Using Bernoulli's equation, $\Delta p$ is related to $\gamma_{a}\left(x_{1}, t\right)$ through

$$
\Delta p\left(x_{1}, t\right)=-\rho_{0}\left[\frac{\partial}{\partial t} \int_{-a}^{x_{1}} \gamma_{a}(s, t) \mathrm{d} s+U \gamma_{a}\left(x_{1}, t\right)\right],
$$

where $\rho_{0}$ denotes the mean fluid density.

The motion of the incident vortex $\Gamma$ is governed by the equation

$$
\frac{\mathrm{d} z_{\Gamma}}{\mathrm{d} t}=W_{\Gamma}^{*}
$$

where an asterisk marks the complex conjugate of a complex number, and

$$
W_{\Gamma}=U-\frac{i}{2 \pi}\left[\int_{-a}^{a} \frac{\gamma_{a}\left(x_{1}, t\right) \mathrm{d} x_{1}}{z_{\Gamma}-x_{1}}+\sum_{k=1}^{n} \frac{\Gamma_{k}}{z_{\Gamma}-z_{\Gamma_{k}}}\right]
$$

is the conjugate flow velocity induced at the instantaneous incident vortex location, excluding its self-singularity. Similarly, the motion of the $\Gamma_{1}, \ldots, \Gamma_{n-1}$ "frozen" trailing edge vortices is governed by

$$
\frac{\mathrm{d} z_{\Gamma_{k}}}{\mathrm{~d} t}=W_{\Gamma_{k}}^{*},
$$

with $k=1, \ldots, n-1$, and

$$
\begin{aligned}
W_{\Gamma_{k}}= & U-\frac{i}{2 \pi}\left[\int_{-a}^{a} \frac{\gamma_{a}\left(x_{1}, t\right) \mathrm{d} x_{1}}{z_{\Gamma_{k}}-x_{1}}+\sum_{\substack{m=1 \\
m \neq k}}^{n} \frac{\Gamma_{m}}{z_{\Gamma_{k}}-z_{\Gamma_{m}}}\right. \\
& \left.+\frac{\Gamma}{z_{\Gamma_{k}}-z_{\Gamma}}\right] .
\end{aligned}
$$

As discussed above, the time-varying position and strength of the $n$th trailing edge vortex are governed by the emended Brown and Michael equation derived by Howe. ${ }^{28}$ In general formulation, the equation is given by

$$
\frac{\mathrm{d} \mathbf{x}_{\Gamma_{n}}}{\mathrm{~d} t} \cdot \nabla \psi_{i}+\frac{\psi_{i}}{\Gamma_{n}} \frac{\mathrm{d} \Gamma_{n}}{\mathrm{~d} t}=\mathrm{v}_{\Gamma_{n}} \cdot \nabla \psi_{i},
$$

where $\Psi_{i}(\mathbf{x}, t)(i=1,2)$ is the stream function corresponding to a flow of unit speed in the $i$-direction at large distances from the airfoil surface, and $\mathrm{v}_{\Gamma_{n}}$ is the velocity at $\Gamma_{n}$ with its local self-potential excluded. For the present case of a thin flat airfoil,

$$
\psi_{1}=\operatorname{Im}\{z\} \text { and } \psi_{2}=\operatorname{Im}\left\{-i \sqrt{z^{2}-a^{2}}\right\} .
$$

Substituting Eq. (10) into Eq. (9) yields the complex form of the Brown and Michael equation in our setup,

$$
\frac{\mathrm{d} z_{\Gamma_{n}}^{*}}{\mathrm{~d} t}+\left(z_{\Gamma_{n}}^{*}-\frac{\operatorname{Re}\left\{a^{2} \sqrt{z_{\Gamma_{n}}^{2}-a^{2}}\right\}}{\operatorname{Re}\left\{z_{\Gamma_{n}}^{*} \sqrt{z_{\Gamma_{n}}^{2}-a^{2}}\right\}}\right) \frac{1}{\Gamma_{n}} \frac{\mathrm{d} \Gamma_{n}}{\mathrm{~d} t}=W_{\Gamma_{n}},
$$

where $W_{\Gamma_{n}}$ is given by Eq. (8) with $k=n$. The strength $\Gamma_{n}$ of the $n$th trailing edge vortex is governed by the Kelvin theorem,

$$
\Gamma_{n}(t)=-\int_{-a}^{a} \gamma_{a}\left(x_{1}, t\right) \mathrm{d} x_{1}-\sum_{k=1}^{n-1} \Gamma_{k}
$$

which ensures that the total system circulation (excluding the incident vortex) vanishes at all times.

The nonlinear system of equations (1)-(8) and (11)-(12) formulates the dynamical problem to be solved together with initial conditions for the vortex location and flap angle and angular velocity. The problem is complemented by the Kutta condition at the airfoil trailing edge,

$$
\gamma_{a}(a, t)=0
$$

requiring that the fluid velocity at the flap downstream end is finite. We assume that release of the first trailing edge vortex starts at $t=0$; the system evolution is then followed for $t>0$ via numerical integration. Details regarding the numerical procedure and analysis are given in Sec. IV.

\section{FAR-FIELD ACOUSTIC RADIATION}

In the small-amplitude, low-Mach, and high-Reynoldsnumber flow setup considered, the far-field acoustic pressure is governed by the Powell-Howe acoustic analogy, ${ }^{22,23}$

$$
\left(\frac{1}{c_{0}^{2}} \frac{\partial^{2}}{\partial t^{2}}-\nabla^{2}\right) p=\rho_{0} \frac{\mathrm{d} v_{f}}{\mathrm{~d} t} \delta\left(x_{2}\right)+\rho_{0} \nabla \cdot(\Omega \times \mathrm{V}),
$$

where $c_{0}$ is the speed of sound, $v_{f}=\partial h / \partial t$ is the normal flap velocity [see (2)], $\delta$ is the Dirac delta function, $\mathbf{V}$ is the fluid velocity, and $\Omega$ is the vector of fluid vorticity. The latter is given by the sum of

$$
\begin{aligned}
& \Omega_{\Gamma}=\hat{\mathrm{x}}_{3} \Gamma \delta\left[\mathrm{x}-\mathrm{x}_{\Gamma}(t)\right] \text { and } \\
& \Omega_{w}=\sum_{k=1}^{n} \Omega_{\Gamma_{k}}=\hat{\mathrm{x}}_{3} \sum_{k=1}^{n} \Gamma_{k} \delta\left[\mathrm{x}-\mathrm{x}_{\Gamma_{k}}(t)\right],
\end{aligned}
$$

which mark the incident and trailing edge wake vorticities, respectively. Combining Eqs. (14) and (15), the acoustic pressure can be written as a sum of "flap motion," "incident vortex," and "wake" contributions,

$$
p(\mathrm{x}, t)=p_{f}(\mathrm{x}, t)+p_{\Gamma}(\mathrm{x}, t)+p_{w}(\mathrm{x}, t),
$$

where

$$
\begin{aligned}
& p_{f}(\mathrm{x}, t)=\rho_{0} \frac{\partial}{\partial t} \int_{0}^{\infty} v_{f}(\tau) \oint_{\mathcal{S}_{f}} G(\mathrm{x}, \mathrm{y}, t-\tau) \mathrm{d} S(\mathrm{y}) \mathrm{d} \tau \\
& p_{\Gamma}(\mathrm{x}, t)=-\rho_{0} \int_{0}^{\infty} \int_{\mathcal{V}_{\Gamma}}\left(\Omega_{\Gamma} \times V_{\Gamma}\right) \cdot \frac{\partial G}{\partial \mathrm{y}}(\mathrm{x}, \mathrm{y}, t-\tau) \mathrm{dyd} \tau
\end{aligned}
$$

and

A. Manela and L. Huang: Sound generated by vortex-flap interaction 


$$
\begin{aligned}
p_{w}(\mathrm{x}, t)=- & \rho_{0} \sum_{k=1}^{n} \int_{0}^{\infty} \int_{\mathcal{V}_{\Gamma_{k}}}\left(\Omega_{\Gamma_{k}} \times \mathrm{V}_{\Gamma_{k}}\right) \\
& \cdot \frac{\partial G}{\partial y}(\mathrm{x}, \mathrm{y}, t-\tau) \mathrm{dyd} \tau .
\end{aligned}
$$

In Eqs. (17)-(19), $\mathcal{S}_{f}$ is the flap surface, $\mathcal{V}_{\Gamma}$ and $\mathcal{V}_{\Gamma_{k}}$ denote the fluid regions occupied by the incident and trailing edge vortices, respectively, $G(\mathbf{x}, \mathbf{y}, t-\tau)$ is the acoustic Green's function having a vanishing normal derivative on the undisturbed airfoil, and $\mathbf{V}_{\Gamma}$ and $\mathbf{V}_{\Gamma_{k}}$ are the velocities of incident and trailing edge vortices, respectively.

We consider a case where the airfoil is acoustically compact. We therefore assume that $a / \lambda \ll 1$, where $\lambda=2 \pi c_{0} / \omega$ is the dimensional acoustic wavelength, with $\omega$ characterizing the frequency of flap oscillations [see Eq. (25)]. The condition for airfoil compactness is then given by $a / \lambda=M(\omega a / 2 \pi U)$ $\ll 1$, where $M=U / c_{0}$ is the mean stream Mach number. This restriction is in accordance with the low Mach assumption set in Eq. (14). In the present two-dimensional configuration, we make use of the far-field two-dimensional compact Green's function, ${ }^{22,23}$

$$
G(\mathrm{x}, \mathrm{y}, t-\tau) \approx \frac{\mathrm{x} \cdot \mathrm{Y}}{2 \pi \sqrt{2 c_{0}}|\mathrm{x}|^{3 / 2}} \frac{\partial}{\partial t}\left\{\frac{H\left(t_{r}-\tau\right)}{\sqrt{t_{r}-\tau}}\right\}, \quad|\mathrm{x}| \rightarrow \infty,
$$

where $\mathbf{Y}(\mathbf{y})$ marks the Kirchhoff vector for the airfoil, and $t_{r}=t-|\mathbf{x}| / c_{0}$ is the acoustic retarded time. We approximate $\mathbf{Y}(\mathbf{y})$ by the Kirchhoff vector for an infinite strip,

$$
\mathrm{Y}(\mathrm{y})=\left\{y_{1}, \operatorname{Re}\left[-\mathrm{i} \sqrt{\left(y_{1}+\mathrm{i} y_{2}\right)^{2}-a^{2}}\right]\right\} .
$$

Starting with evaluation of $p_{f}(\mathbf{x}, t)$, substitute Eq. (20) into Eq. (17) together with Eq. (2). The space integral can be calculated explicitly to yield

$$
\begin{aligned}
p_{f}(\mathrm{x}, t) \approx & \frac{\rho_{0} a^{3} \cos \alpha}{2 \pi \sqrt{2 c_{0}|\mathrm{x}|}}\left[\frac{2}{3}\left(1-\bar{\eta}^{2}\right)^{3 / 2}\right. \\
& \left.-\bar{\eta}\left(\pi-\phi_{\bar{\eta}}+\frac{\sin 2 \phi_{\bar{\eta}}}{2}\right)\right] \frac{\partial^{2}}{\partial t^{2}} \int_{0}^{t r} \frac{\mathrm{d} \theta / \mathrm{d} \tau}{\sqrt{t_{r}-\tau}} \mathrm{d} \tau,
\end{aligned}
$$

where $\cos \alpha=x_{2} /|\mathbf{x}|$ indicates the observer direction, and $\phi_{\eta}=\arccos (-\bar{\eta})$. To evaluate $p_{\Gamma}$, substitute Eq. (20) together with Eq. (15) into Eq. (18) to obtain

$$
\begin{aligned}
p_{\Gamma}(\mathrm{x}, t) \approx & \frac{\rho_{0} \Gamma \sin \alpha}{2 \pi \sqrt{2 c_{0}|\mathrm{x}|}} \frac{\partial}{\partial t} \int_{0}^{t r} \frac{V_{\Gamma}^{(2)}(\tau) \mathrm{d} \tau}{\sqrt{t_{r}-\tau}} \\
& -\frac{\rho_{0} \Gamma \cos \alpha}{2 \pi \sqrt{2 c_{0}|\mathrm{x}|}} \frac{\partial}{\partial t} \\
& \times \int_{0}^{t r}\left[V_{\Gamma}^{(1)}(\tau) \frac{\partial Y_{2}}{\partial y_{2}}-V_{\Gamma}^{(2)}(\tau) \frac{\partial Y_{2}}{\partial y_{1}}\right]_{x_{\Gamma}(\tau)} \frac{\mathrm{d} \tau}{\sqrt{t_{r}-\tau}},
\end{aligned}
$$

where $V_{\Gamma}^{(j)}$ denotes the velocity component of the incident vortex in the $x_{j}$-direction. Similarly, $p_{w}$ is evaluated by substituting Eq. (20) and Eq. (15) into Eq. (19), to yield

$$
\begin{aligned}
p_{w}(\mathrm{x}, t) \approx & \sum_{k=1}^{n}\left\{\frac{\rho_{0} \Gamma_{k} \sin \alpha}{2 \pi \sqrt{2 c_{0}|\mathrm{x}|}} \frac{\partial}{\partial t} \int_{0}^{t r} \frac{V_{\Gamma_{k}}^{(2)}(\tau) \mathrm{d} \tau}{\sqrt{t_{r}-\tau}}\right. \\
& -\frac{\rho_{0} \Gamma_{k} \cos \alpha}{2 \pi \sqrt{2 c_{0}|\mathrm{x}|}} \frac{\partial}{\partial t} \\
& \left.\times \int_{0}^{t r}\left[V_{\Gamma_{k}}^{(1)}(\tau) \frac{\partial Y_{2}}{\partial y_{2}}-V_{\Gamma_{k}}^{(2)}(\tau) \frac{\partial Y_{2}}{\partial y_{1}}\right]_{x_{\Gamma_{k}}(\tau)} \frac{\mathrm{d} \tau}{\sqrt{t_{r}-\tau}}\right\}
\end{aligned}
$$

where $V_{\Gamma_{k}}^{(j)}$ marks the $x_{j}$-velocity component of the vortex $\Gamma_{k}$. Note that, unlike the "lift-type" dipole found for $p_{f}$ in Eq. (22) (proportional to $\cos \alpha$, therefore directed along the $x_{2}$-axis), both dipoles in Eq. (23) and Eq. (24) have a "suction-type" component oriented parallel to the mean-flow $x_{1}$-direction (proportional to $\sin \alpha$ ). This difference results directly from non-linear vortex-airfoil interactions, leading to velocity components of the incident and trailing edge vortices in the normal $x_{2}$-direction. Consequently, dipole-type sound is radiated in the $x_{1}$-direction, reflecting both incident vortex motion at times when the vortex passes close to the airfoil [in which $V_{\Gamma}^{(2)} \neq 0$ ], and flap motion causing trailing edge vortices to shift in the $x_{2}$-direction [having $V_{\Gamma_{k}}^{(2)} \neq 0$ ].

\section{SCALING AND ANALYSIS}

To non-dimensionalize the problem, the length, velocity, time, and pressure are scaled by $a, U, a / U$ and $\rho_{0} U^{2}$, respectively. Omitting presentation of the full non-dimensional problem for brevity, we note the scaled form of the equation of motion for the flap [the non-dimensional counterpart of Eq. (3)] for later reference,

$$
\frac{\mathrm{d}^{2} \theta}{\mathrm{d} \bar{t}^{2}}+\bar{\omega}^{2} \theta=\bar{\beta} \int_{\bar{\eta}}^{1} \Delta \bar{p}\left(\bar{x}_{1}, \bar{t}\right)\left(\bar{x}_{1}-\bar{\eta}\right) \mathrm{d} \bar{x}_{1} .
$$

Here the non-dimensional variables are marked by overbars, and the equation is governed by the parameters

$$
\bar{\eta}, \bar{\omega}=\sqrt{\frac{k_{\theta} a^{2}}{I_{f} U^{2}}} \text { and } \bar{\beta}=\frac{\rho_{0} a^{4}}{I_{f}},
$$

with the latter two denoting the system natural frequency and fluid-loading number, respectively. Effectively, $\bar{\omega}$ may be regarded as an equivalent Strouhal number for the system, while $\bar{\beta}$ determines the relative effect of fluid loading on flap angular motion. In addition to Eq. (26), the dynamical problem is also governed by the parameters

$$
\bar{\Gamma}=\frac{\Gamma}{2 \pi a U} \text { and } \overline{\mathbf{x}}_{\Gamma}(0)=\frac{\mathbf{x}_{\Gamma}(0)}{a},
$$

specifying the scaled incident vortex circulation and initial location, respectively. To illustrate our results, we focus on a case of an incident vortex with $\bar{\Gamma}=0.2$, initially located at $\overline{\mathbf{x}}_{\Gamma}(0)=(-20,0.2)$, sufficiently far upstream of the airfoil, where it essentially convects along a straight line with the mean flow. In addition, we fix $\bar{\eta}=0.8$, which corresponds to a trailing edge flap capturing $10 \%$ of the airfoil chord. The 
remaining free parameters are therefore the system natural frequency $\bar{\omega}$ and fluid-loading number $\bar{\beta}$, the effects of which will be studied below. As an order of magnitude approximation, for a $1 \mathrm{~cm}$ thick aluminum airfoil of length $0.5 \mathrm{~m}(a=0.25 \mathrm{~m})$ subject to air flow, we find that $\bar{\beta} \approx 4.4$ when $\bar{\eta}=0.8$. Considering, in addition, a torsion spring with constant $k_{\theta}=2 \mathrm{Nt} / \mathrm{rad}$ (per unit span) and a wind speed of $U=10 \mathrm{~m} / \mathrm{s}$, we obtain $\bar{\omega} \approx 1$. We therefore focus on cases where $\bar{\omega}, \bar{\beta} \sim O(1)$. Airfoil dimensions and materials given above are typical of standard high-lift devices used in commercial transport vehicles and may vary with reducing the scale to consider micro air vehicle applications. Nevertheless, our calculations indicate that no significant qualitative changes are observed in our results when considering other (larger or smaller) values of $\bar{\omega}$ and $\bar{\beta}$.

Numerical solution of the dynamical problem requires discretization of the system of equations (1)-(8) and (11)-(13) in both space (along the airfoil chord) and time (from $t=0$ to a final time). Space discretization is needed to express the vorticity distribution $\gamma_{a}\left(x_{1}, t\right)$ along the airfoil. In a manner described in the Appendix, we expand $\bar{\gamma}_{a}\left(\bar{x}_{1}, \bar{t}\right)$ $=\gamma_{a} / U$ in a Fourier-type series, which simplifies the formulation of impermeability condition (1). Apart from specifying the initial incident vortex location, two initial conditions are required for the flap state, namely, $\theta(0)=\mathrm{d} \theta / \mathrm{d} t(0)=0$, as mentioned in the beginning of Sec. II. We thus study a case of a "passive" (non-actuated) flap, the motion of which is initiated by the passing vortex loading, as reflected through the right-hand side of Eq. (25).

The system of equations is integrated in time using a fourth-order Runge-Kutta algorithm. The typical time step used for integration was $\pi /(500 \bar{\omega}$ ) (and not larger than $\sim \pi / 500$ for $\bar{\omega}<1$ ) along most of the integration interval, which proved sufficient for convergence (with errors $0.1 \%$ ). Particular care was required at times when the vortex passes above airfoil leading and trailing edges: at these times, smaller time steps $[\sim \pi /(3000 \bar{\omega})$ and not larger than $\sim \pi / 3000$ for $\bar{\omega}<1]$ were needed to achieve a converged result for the acoustic signal, where sharp time-variations were observed (see Sec. V). Yet, it is worthwhile to mention that the running time required for a single computation (that is, calculation of system evolution for a specific set of parameters from an initial state, when the vortex is located far upstream of the airfoil, to a time after it has passed past the flap trailing edge) was markedly short, lasting only few minutes on a standard desktop machine. To validate convergence of our numerical solution, test runs were carried out at space and time discretizations smaller than those indicated above, and yielded results within a $\sim 0.1 \%$ difference, for both dynamical and acoustic calculations.

Adopting the scaling introduced, the non-dimensional form of the acoustic pressure (16) is

$$
\begin{aligned}
\frac{p(\mathrm{x}, t)}{\rho_{0} U^{2}} & =\sqrt{\frac{M}{8|\overline{\mathrm{x}}|}} \Pi_{\mathrm{tot}}\left(\bar{t}_{r}\right) \\
& =\sqrt{\frac{M}{8|\overline{\mathrm{x}}|}}\left[\Pi_{f}\left(\bar{t}_{r}\right)+\Pi_{\Gamma}\left(\bar{t}_{r}\right)+\Pi_{w}\left(\bar{t}_{r}\right)\right],
\end{aligned}
$$

where

$$
\begin{aligned}
\Pi_{f}\left(\bar{t}_{r}\right) \approx & \frac{1}{\pi} \cos \alpha\left[\frac{2}{3}\left(1-\bar{\eta}^{2}\right)^{3 / 2}\right. \\
& \left.-\bar{\eta}\left(\pi-\phi_{\bar{\eta}}+\frac{\sin 2 \phi_{\bar{\eta}}}{2}\right)\right] \frac{\partial^{2}}{\partial \bar{t}^{2}} \int_{0}^{\bar{t}_{r}} \frac{\mathrm{d} \theta / \mathrm{d} \tau}{\sqrt{\bar{t}_{r}-\tau}} \mathrm{d} \tau \\
\Pi_{\Gamma}\left(\bar{t}_{r}\right) \approx & 2 \bar{\Gamma}\left\{\sin \alpha \frac{\partial}{\partial \bar{t}} \int_{0}^{\bar{t}_{r}} \frac{\bar{V}_{\Gamma}^{(2)} \mathrm{d} \tau}{\sqrt{\bar{t}_{r}-\tau}}\right. \\
& \left.-\cos \alpha \frac{\partial}{\partial \bar{t}} \int_{0}^{\bar{t}_{r}} \frac{\left[\bar{V}_{\Gamma}^{(1)}+i \bar{V}_{\Gamma}^{(2)}\right] \bar{z}_{\Gamma} \mathrm{d} \tau}{\sqrt{\left(\bar{z}_{\Gamma}^{2}-1\right)\left(\bar{t}_{r}-\tau\right)}}\right\}
\end{aligned}
$$

and

$$
\begin{aligned}
\Pi_{w}\left(\bar{t}_{r}\right) \approx & \sum_{k=1}^{n} 2 \bar{\Gamma}_{k}\left\{\sin \alpha \frac{\partial}{\partial \bar{t}} \int_{0}^{\bar{t}_{r}} \frac{\bar{V}_{\Gamma_{k}}^{(2)} \mathrm{d} \tau}{\sqrt{\bar{t}_{r}-\tau}}\right. \\
& \left.-\cos \alpha \frac{\partial}{\partial \bar{t}} \int_{0}^{\bar{t}_{r}} \frac{\left[\bar{V}_{\Gamma_{k}}^{(1)}+i \bar{V}_{\Gamma_{k}}^{(2)}\right] \bar{z}_{\Gamma_{k}} \mathrm{~d} \tau}{\sqrt{\left(\bar{z}_{\Gamma_{k}}^{2}-1\right)\left(\bar{t}_{r}-\tau\right)}}\right\} .
\end{aligned}
$$

In (31), $\bar{\Gamma}_{k}=\Gamma_{k} /(2 \pi a U)$. The results for the acoustic pressure will be presented in terms of the acoustic "kernels" $\Pi_{\mathrm{tot}}, \Pi_{f}$, $\Pi_{\Gamma}$, and $\Pi_{w}$. Note that the non-dimensional acoustic pressure is governed, in addition to the parameters given by Eqs. (26) and (27), by the observer direction $\alpha=\cos ^{-1}\left(x_{2} /|\mathbf{x}|\right)$.

\section{NUMERICAL RESULTS}

Figure 2 presents the flap motion and vortical field induced by the passage of the incident vortex above the airfoil at $\bar{\omega}=1$ and $\bar{\beta}=10$. The solid lines in Figs. 2(a) and 2(b) show time variations of flap angle $\theta$ and total airfoil circulation $\Gamma_{a} / a U$ [see Eq. (32)], respectively, and Figs. 2(c) and $2(\mathrm{~d})$ present time snapshots of the vortical flow-field at $U t / a=20$ and $U t / a=40$, respectively. For easy reference, the dash-dotted lines in Figs. 2(a) and 2(b) confine the time interval during which the incident vortex passes above the airfoil, and the dotted and dashed lines depict variations of flap angle and airfoil circulation in non-flapped $(\bar{\beta}=0)$ and torsion-free $(\bar{\omega}=0)$ configurations, respectively. Solid and dashed curves in Figs. 2(c) and 2(d) mark trajectories of the incident vortex $\Gamma$ and trailing edge vortex $\Gamma_{2}$, respectively, and bold solid lines show airfoil location. Physically, the torsion-free case appearing in Figs. 2(a) and 2(b) represents a "quasi-static" limit of the problem, where the residence time of the vortex above the airfoil is so short that the flap cannot adjust its equilibrium position during vortex passage. Applying a similar interpretation, the case $\bar{\omega}=1$ matches a configuration where the vortex residence time above the airfoil equals the mechanical period of flap motion.

In accordance with Kelvin's theorem (12), the total airfoil circulation plotted in Fig. 2(b) is given by

$$
\frac{\Gamma_{a}}{a U}=\int_{-1}^{1} \bar{\gamma}_{a}\left(\bar{x}_{1}, \bar{t}\right) \mathrm{d} \bar{x}_{1}=-\sum_{k=1}^{n} \frac{\Gamma_{k}}{a U},
$$


ensuring that the total system circulation is preserved at all times. Tracking the system evolution from $t=0$ (where $\Gamma_{a}=0$ ), we observe that at early times (prior to incident vortex passage above the airfoil), the incident vortex has a relatively minor impact on flap motion, inducing vanishingly small flap oscillations [see the solid line in Fig. 2(a) for $U t / a<15]$. This is accompanied by release of trailing edge vortex $\Gamma_{1}>0$, together with a monotonic decrease in airfoil circulation [solid line in Fig. 2(b)]. Shortly after the incident vortex passes above the airfoil leading edge, $\mathrm{d} \Gamma_{1} / \mathrm{d} t$ changes sign, and $\Gamma_{1}$ is detached from the airfoil trailing edge. At that time, marked by the first cross from left in Fig. 2(b), release of $\Gamma_{2}<0$ is initiated [see Fig. 2(c)]. During passage of the incident vortex above the airfoil, a sharp increase in the airfoil circulation is observed, related directly to a decrease in $\Gamma_{2}(t)$ [see Eq. (32)], and significant flap deflection occurs. Remarkably, soon after the incident vortex has passed above the airfoil trailing edge, $\Gamma_{2}$ is detached from the flap end point [see the triangle symbol adjacent to the right dash-dotted line in Fig. 2(b)], and forms a "vortex pair" motion together with $\Gamma$. Simultaneously, subsequent release of trailing edge vortices continues, in the form of a "vortex street" which reflects the oscillatory flap motion [see Fig. 2(d)]. In accordance with the small-amplitude assumption set for the analysis of flap motion (see Sec. II), $|\theta(t)| \lesssim 10^{\circ}$ at all times. As will be demonstrated below, flap motion amplitude is affected significantly by $\bar{\omega}$ and $\bar{\beta}$, and reduces monotonically with decrease in the latter.

To elucidate the impact of flap motion on the fluid dynamical behavior, the dashed and dotted lines in Figs. 2(a) and 2(b) present flap angular deflection and total airfoil circulation for quasi-static $(\bar{\omega}=0)$ and non-flapped $(\bar{\beta}=0)$ setups, respectively. In the quasi-static configuration, the spring counter-effect to balance fluid loading moment is absent, and flap motion is governed merely by flap inertia and pressure jump loading effects [see Eq. (25)]. Subsequently, this setup is characterized by relatively large flap deflections: as the counterclockwise vortex approaches the airfoil it pulls the flap upwards, and then, as it convects above the flap and passes the airfoil trailing edge, it pushes the flap away. This simple "passive-motion" mechanism reduces the singularity of incident vortex interaction with airfoil trailing edge,
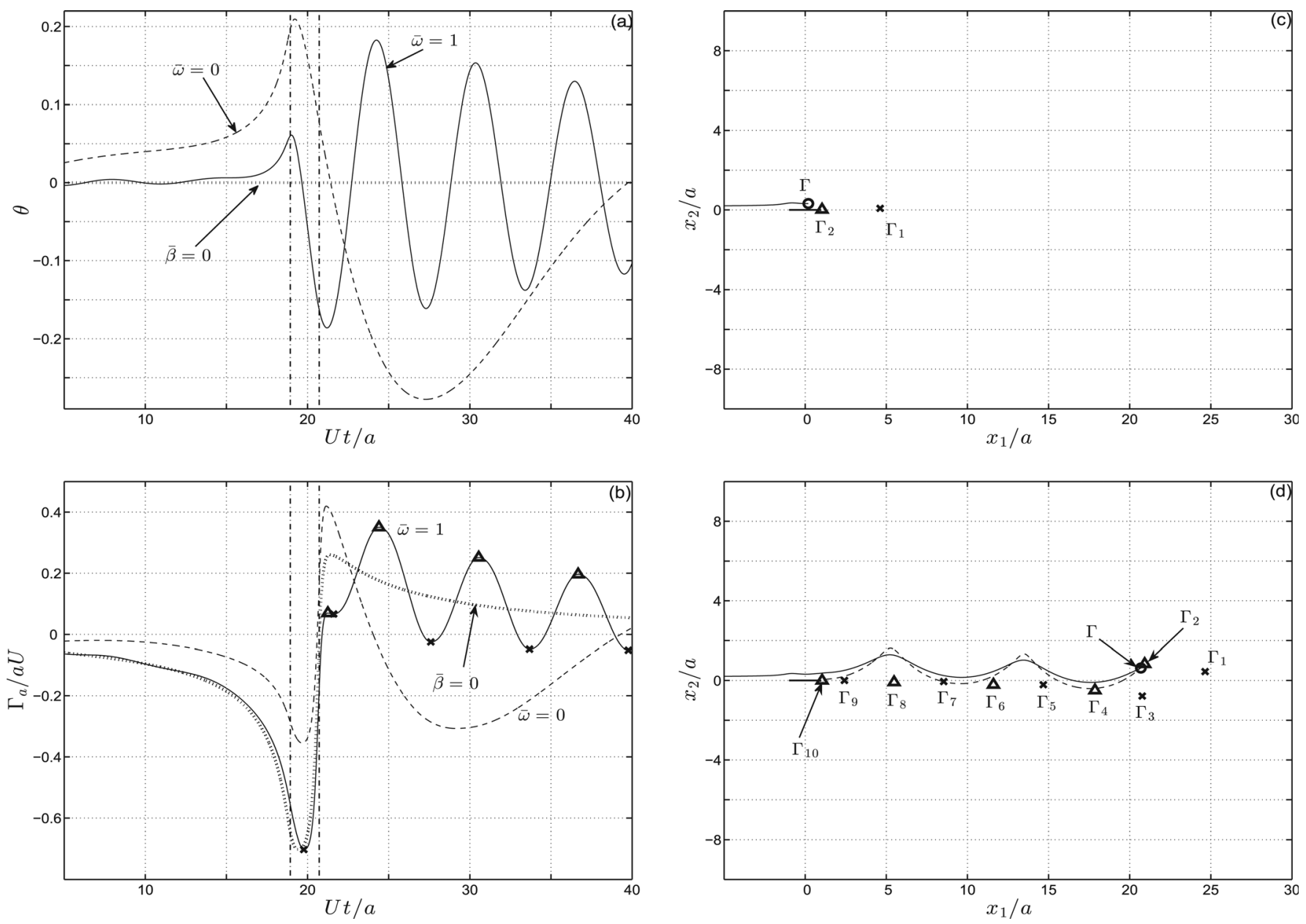

FIG. 2. Flap motion and vortical field induced by a passing vortex with $\bar{\omega}=1$ and $\bar{\beta}=10$ : (a) flap angle (solid line); (b) total airfoil circulation (solid line); (c) and (d) time-snapshots of incident vortex (circle) and trailing edge vortices (crosses and triangles) locations at times $U t / a=20[(\mathrm{c})]$ and $U t / a=40[(\mathrm{~d})]$. Crosses and triangles denote trailing edge vortices with positive and negative circulations, respectively. Solid and dashed curves show trajectories of incident vortex and vortex $\Gamma_{2}$, respectively. Bold solid lines in (c) and (d) indicate airfoil location. In (a) and (b), vertical dash-dotted lines confine time interval during which incident vortex passes above the airfoil. In (b), crosses and triangles mark time instants in which trailing edge vortices $\Gamma_{1}, \ldots, \Gamma_{9}$ are detached, ordered from left to right. In (a) and (b), Dashed and dotted lines show flap angle and total airfoil circulation for quasi-static $(\bar{\omega}=0)$ and non-flapped $(\bar{\beta}=0)$ configurations, respectively. 
which, in turn, causes reduction in sound radiation at trailing edge time (see Fig. 4). Turning to the rigid airfoil configuration $[\bar{\beta}=0$; see the dotted lines in Figs. 2(a) and 2(b)], we note that at early times $(U t / a<15)$ the differences between airfoil circulations in the dotted and solid lines are minor. However, the effect of induced flap oscillations, reflected by the wavy variation of the solid curve for $U t / a>20$, is absent in both non-flapped and torsion-free setups. In fact, as has been demonstrated in previous studies (e.g., Ref. 29), the trailing edge wake in a stationary-airfoil setup can be described using only three trailing edge vortices, since airfoil circulation at late times is monotonically decreasing. This is in marked difference from the present vortical-flow description, where vortex-street vortices are generated at late times [see Fig. 2(d)]. These vortices affect the system acoustic signature, as will be demonstrated in Figs. 3 and 5.

Focusing on Fig. 2(a), we observe that induced flap oscillations, shown by the solid line, are characterized by the respective system natural frequency, $\bar{\omega}=1$. In addition, our calculations indicate that the system response to incident-vortex forcing at other values of $\bar{\omega}$ seemingly results in flap oscillations at the corresponding frequency. This can be rationalized by recalling that the forcing vortex, mathematically described as a propagating delta function, contains the entire frequency spectrum. As the vortex passes above the airfoil, the flap-fluid system "chooses" to amplify its natural frequency component above all other frequencies. Yet, while it is the forcing term in Eq. (25) that initially excites flap motion, it is the same mechanism that acts to damp oscillations at later times. At $U t / a \gg 1$ (not shown here for better clarity of the intermediate time domain), flap motion, as well as release of trailing edge vortices, vanish. As will be demonstrated in Fig. 5, both effects of oscillation excitation and damping become more pronounced with increasing $\bar{\beta}$. Viscous effects, not included in the present potential-flow analysis, are expected to augment the effect of fluid damping further.

Figure 3 shows the far-field acoustic radiation in the $x_{2}-$ direction [Figs. 3(a) and 3(c)] and $x_{1}$-direction [Figs. 3(b) and 3(d)] for the same parameter combination as in Fig. 2, $\bar{\omega}=1$ and $\bar{\beta}=10$. Figures 3(a) and 3(b) present decompositions of the acoustic signal into its flap motion, incident vortex, and wake sound components [see (28)-(31)], and Figs. 3(c) and 3(d) depict the total acoustic signature. The dashed vertical lines, as in Fig. 2, mark the time interval during which the incident vortex passes above the airfoil. To
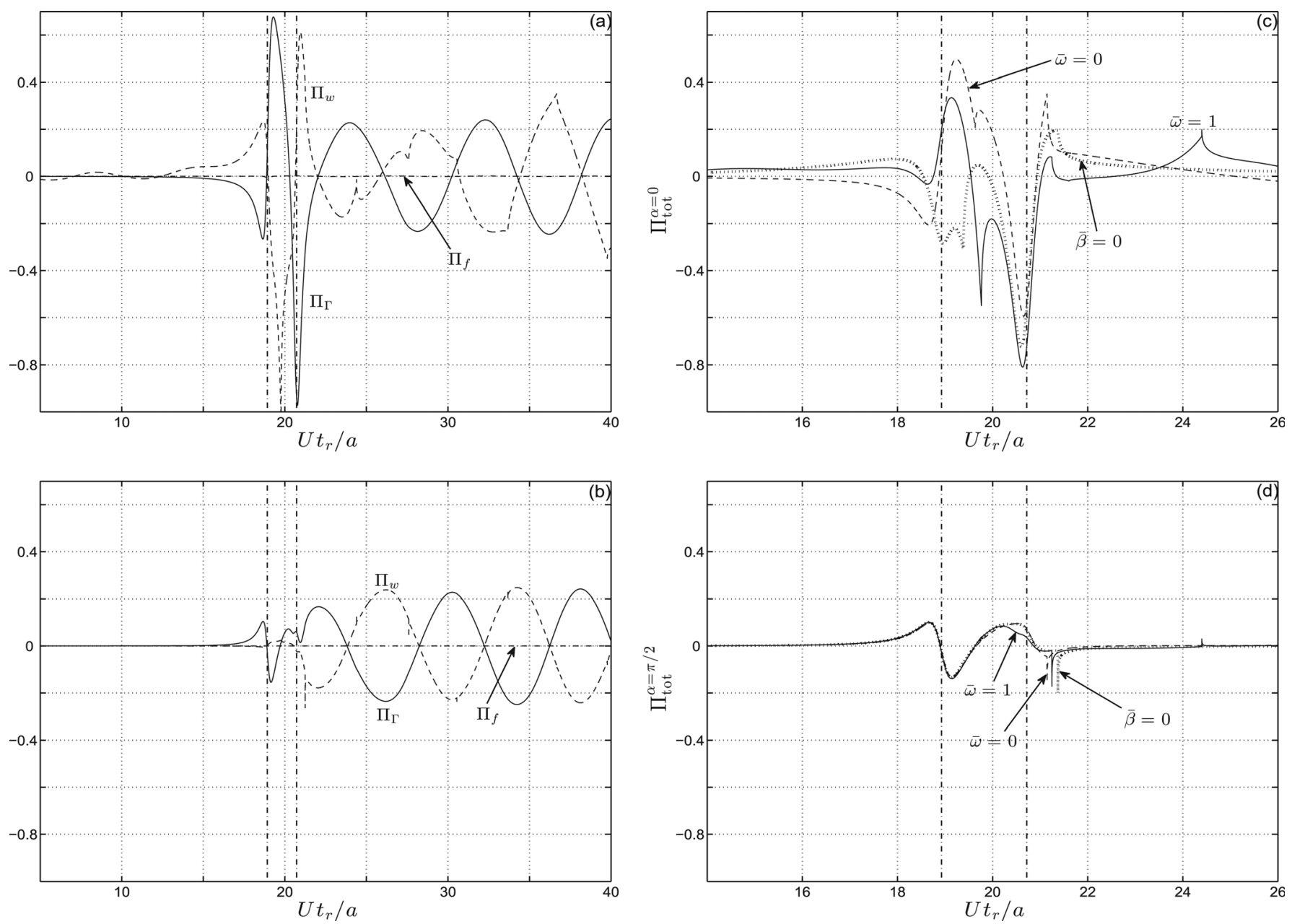

FIG. 3. Far-field acoustic radiation in the directions normal $\alpha=0$ [(a) and (c)] and parallel $\alpha=\pi / 2$ [(b) and (d)] to the airfoil for $\bar{\omega}=1$ and $\bar{\beta}=10$ : (a) and (b) separate contributions of incident vortex $\left(\Pi_{\Gamma}\right)$, wake $\left(\Pi_{w}\right)$ and flap-motion $\left(\Pi_{f}\right)$ radiations; (c) and (d) total acoustic signals (solid lines). Vertical dash-dotted lines confine time interval during which the incident vortex passes above the airfoil. In (c) and (d), dashed and dotted lines mark total acoustic signatures for quasi-static $(\bar{\beta}=10, \bar{\omega}=0)$ and non-flapped $(\bar{\beta}=0)$ configurations, respectively. 

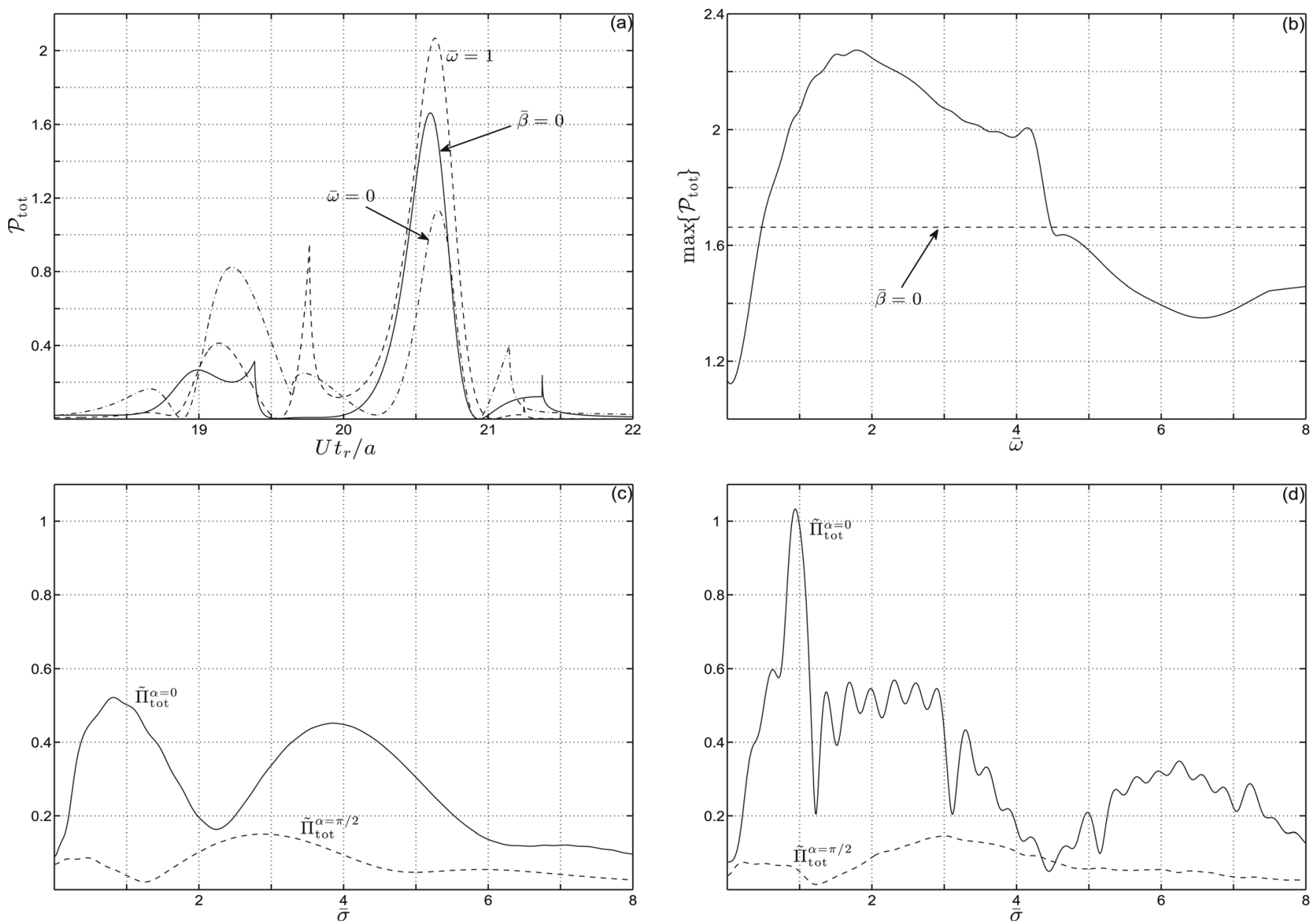

FIG. 4. Effect of system natural frequency $\bar{\omega}$ on sound energy amplitude $\mathcal{P}_{\text {tot }}$ for $\bar{\beta}=10$ : (a) comparison between $\mathcal{P}_{\text {tot }}$ for $\bar{\omega}=0$ (quasi static), $\bar{\omega}=1$, and $\bar{\beta}=0$ (non-flapped) configurations; (b) variation of maximum $\mathcal{P}_{\text {tot }}$, achieved at trailing edge time, with $\bar{\omega}$; (c) and (d) time Fourier decompositions [defined in Eq. (34)] of total lift $\left(\tilde{\Pi}_{\text {tot }}^{\alpha=0}\right)$ and suction $\left(\tilde{\Pi}_{\text {tot }}^{\alpha=\pi / 2}\right)$ dipoles for non-flapped [(c)] and $\bar{\omega}=1[(\mathrm{~d})]$ setups. Dashed line in (b) marks the maximum value of $\mathcal{P}_{\text {tot }}$ for $\bar{\beta}=0$.

follow on the comparison made in Fig. 2, the dashed and dotted lines in Figs. 3(c) and 3(d) show the counterpart total radiations in quasi-static $(\bar{\omega}=0)$ and non-flapped $(\bar{\beta}=0)$ setups.

At first we observe that $\Pi_{f} \approx 0$ along both $x_{1^{-}}$and $x_{2^{-}}$ directions. More precisely, for the present level of approximation, neglecting quadrupole and higher-order sound effects, no flap-motion sound is emitted in the $x_{1}$-direction $\left[\Pi_{f}(\alpha=\pi / 2)=0\right.$, see Eq. (29)], while small pressure fluctuations [invisible in the scale of Fig. 3(a)] are radiated in the $x_{2}$-direction. It is therefore vortex sound which dominates the far-field acoustic signal at all times. Focusing on early times, both $\Pi_{\Gamma}$ and $\Pi_{w}$ in Figs. 3(a) and 3(b) are vanishingly small, since the incident vortex at this stage convects along a straight line and the strength of trailing edge vorticity is negligible. However, significant pressure variations are observed as $\Gamma$ approaches the airfoil leading edge and passes above it. This, in turn, has a counter effect on the trailing edge vortex $\Gamma_{1}(t)$, which acts to reduce the total sound radiation [cf. the dashed and solid lines in Fig. 3(a) at $\left.U t_{r} / a \approx 19\right]$. Shortly after, when the vortex passes above the airfoil, $\Gamma_{1}$ is detached from the airfoil trailing edge (with its circulation fixed) and release of $\Gamma_{2}$ is initiated. Similarly to $\Gamma_{1}, \Gamma_{2}$ counteracts to reduce the sound radiated by the incident vortex as it passes above the flap trailing edge. As found in Fig. 2, at later times $\Gamma_{2}$ is detached from the trailing edge, forming a vortex pair together with the incident vortex. From this stage on, pressure fluctuations produced by these counter-rotating vortices completely cancel each other, resulting in a "silent" pair. Late time radiation is therefore attributed solely to trailing edge vortex-street vortices $\Gamma_{3}, \ldots, \Gamma_{n}$ released in response to induced flap oscillations.

Summarizing the above discussion, the total system radiation presented by the solid lines in Figs. 3(c) and 3(d) can be described as a combination of relatively strong leading and trailing edge interactions of the airfoil with the incident vortex (which are, yet, reduced by the impact of trailing edge wake), together with late-time trailing edge vortex sound reflecting the motion of the flap. Interestingly, while flap motion is the indirect cause for late-time radiation, direct flap sound is negligible at all times. The late-time radiation is significantly stronger in the normal direction than in the mean-flow direction, and decays to zero in all directions with the vanishing of flap motion at $U t / a \gg 1$. Comparing between the flap-on-spring (solid), torsion-free (dashed), and non-flapped (dotted) signatures, our calculations indicate that significant late-time radiation occurs only in the flap on a spring configuration. Notably, when focusing on pressure 
levels radiated at trailing edge time (i.e., when the incident vortex passes above airfoil trailing edge), we observe that the flap may have an amplifying (for $\bar{\omega}=1$ ) or absorbing (for $\bar{\omega}=0$ ) effect on the sound compared with the rigid case [cf. the levels of total signatures in the vicinity of the vertical right line in Fig. 3(c)]. This motivates further investigation on the effect of $\bar{\omega}$ on the system acoustic radiation, which will be carried out in Fig. 4. Note, however, that the suction dipole is considerably less affected by the presence of the flap [see Fig. 3(d)].

Figure 4 analyzes the effect of flap-fluid natural frequency $\bar{\omega}$ on the system sound level. Towards this end, Fig. 4(a) presents the far-field sound energy amplitude,

$$
\mathcal{P}_{\mathrm{tot}}\left(\bar{t}_{r}\right)=\int_{0}^{2 \pi} \Pi_{\mathrm{tot}}^{2}\left(\bar{t}_{r}, \alpha\right) \mathrm{d} \alpha
$$

obtained by quadrature of the squared total acoustic pressure (28) over a circle of radius $|\overline{\mathbf{x}}| \rightarrow \infty$. The solid line shows the acoustic amplitude for a non-flapped airfoil $(\bar{\beta}=0)$, and the dashed and dash-dotted lines present the counterpart results for $\bar{\beta}=10$ with $\bar{\omega}=0$ and $\bar{\omega}=1$, respectively. As noted in Fig. 3, we observe that the largest sound energy amplitude is obtained in the proximity of trailing edge time (at $U t_{r} / a \approx 20.7$ ), and thus focus on the effect of $\bar{\omega}$ at that time. This effect is illustrated in Fig. 4(b), where the maximum value of $\mathcal{P}_{\text {tot }}$ is shown as function of $\bar{\omega}$. For reference, the counterpart sound energy amplitude in the case of a rigid airfoil [i.e., the maximum value of the solid line in Fig. 4(a)] is given by the dashed line. In support of Fig. 3, the results confirm that the flap may act as sound "amplifier" or "absorber," depending on the value of system natural frequency: at $0.5 \lessgtr \bar{\omega} \lesssim 4.5$, the flap amplifies sound energy amplitude above the reference rigid-airfoil value, while at lower and larger frequencies the flap attenuates radiation. Note that at large values of $\bar{\omega}$ (not presented here), $\mathcal{P}_{\text {tot }}$ converges to its non-flapped form, as flap oscillations vanish and the airfoil becomes essentially rigid.

To gain further insight into the above results, Figs. 4(c) and 4(d) present time Fourier transforms,

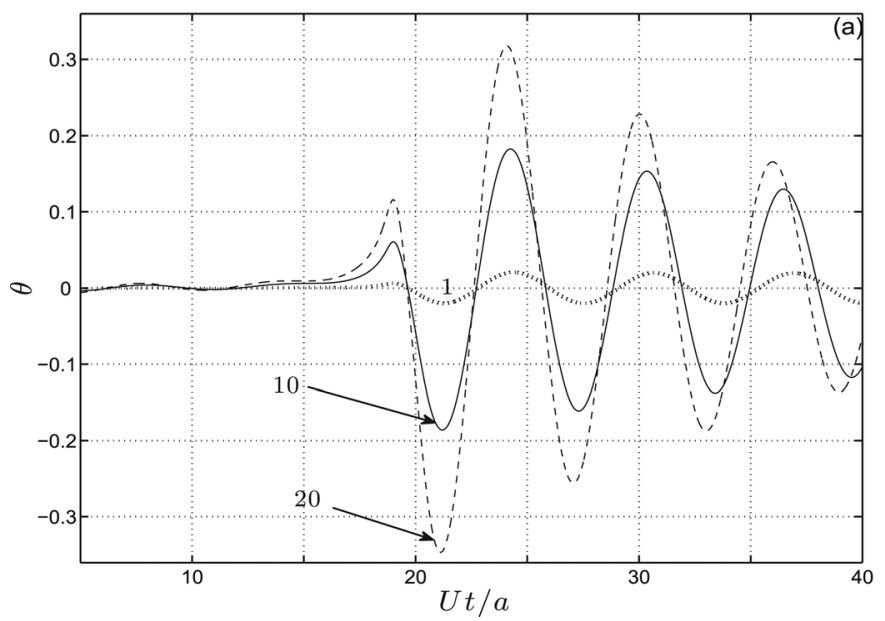

$$
\tilde{\Pi}_{\text {tot }}(\bar{\sigma})=\int_{-\infty}^{\infty} \Pi_{\text {tot }}(\bar{t}) \exp (-i \bar{\sigma} \bar{t}) \mathrm{d} \bar{t}
$$

of the lift ( $\tilde{\Pi}_{\text {tot }}^{\alpha=0}$, solid lines) and suction $\left(\tilde{\Pi}_{\text {tot }}^{\alpha=\pi / 2}\right.$, dashed lines) dipoles for non-flapped [Fig. 4(c)] and flapped $\bar{\omega}=1$ [Fig. 4(d)] airfoils. The plots focus on small and intermediate values of $\bar{\sigma}$. Higher frequencies acquire vanishingly small portion of the signal spectral energy and are therefore not included. Starting with the spectral decomposition of the reference rigid airfoil in Fig. 4(c), we observe that the lift dipole has two distinct maxima near $\bar{\sigma} \approx 0.8$ and $\bar{\sigma} \approx 3.8$, and the less-dominant suction dipole acquires a maximum close to $\bar{\sigma} \approx 3$. In addition, the contribution of the $\bar{\sigma}=0$ component to the lift dipole is the smallest among all frequencies presented. These features are qualitatively reflected in the results of Fig. 4(b). As mentioned in the discussion of Fig. 2(a), flap motion at low frequencies essentially follows the velocity field induced by the incident vortex. The flap is therefore pushed away from the vortex at trailing edge time, a passive motion which reduces its edge interaction effect. At intermediate frequencies [within the range amplified by airfoil-vortex interaction, as presented in Fig. 4(c)], the flap resonates with respective components of the rigid airfoil spectrum, causing amplification of the $\bar{\beta}=0$ reference signal. With further increasing $\bar{\omega}$, the resonance response decays, and the flap spring acts as sound absorber, transferring fluid kinetic energy into small-amplitude oscillatory motion. At very high frequencies, the amplitude of flap motion becomes vanishingly small, and the airfoil recovers its rigid-body signature. A typical spectrum of the system signature at an intermediate $(\bar{\omega}=1)$ frequency is shown in Fig. 4(d), where amplification of the respective $\bar{\sigma}=1 \mathrm{com}-$ ponent is clearly visible.

We conclude the presentation of results by studying the effect of fluid loading number $\bar{\beta}$ on the system dynamical and acoustic response. Given the non-dimensional formulation in Eq. (25), it is expected that increase in the value of $\bar{\beta}$ would magnify the coupling between flap and fluid motions. Figure 5 presents the variation with $\bar{\beta}$ of flap angle [Fig. 5(a)] and total acoustic radiation in the $x_{2}$-direction [Fig. 5(b)] for

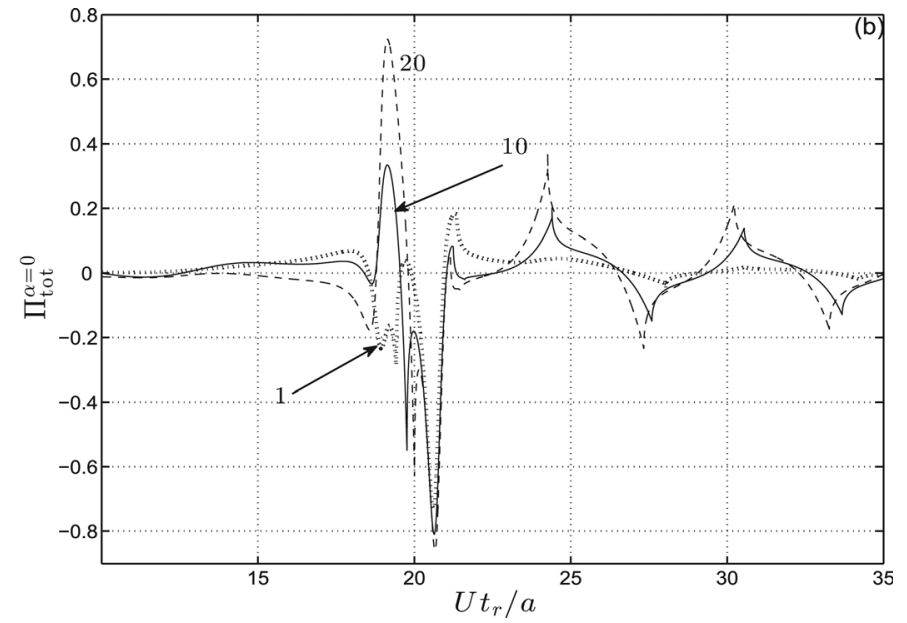

FIG. 5. Effect of fluid-loading number $\bar{\beta}$ on (a) flap angle and (b) far-field radiation in the direction $\alpha=0$ normal to the wing, for $\bar{\omega}=1$. Numbers denote respective values of $\bar{\beta}$. 
$\bar{\omega}=1$. Solid lines show time-variations for $\bar{\beta}=10$ [identical with those presented in Figs. 2(a) and 3(c)], and dotted and dashed lines mark counterpart results for $\bar{\beta}=1$ and $\bar{\beta}=20$, respectively. The two-fold effect of increasing $\bar{\beta}$ on flap motion is clearly observed: namely, initiation of significant flap oscillations (growing with $\bar{\beta}$ ) when the incident vortex passes above the airfoil; and damping of these oscillations at later times. Figure 5(b) reflects similar behavior in the farfield pressure: with increasing $\bar{\beta}$, vortex-airfoil interaction becomes more intense, leading to higher sound levels at incident vortex interaction with airfoil end points. In addition, early- and late-time radiations are observed for $\bar{\beta} \gg 1$, resulting from the contribution of wake-sound component $\Pi_{w}$. As noted in Fig. 3, direct flap noise $\Pi_{f}$ remains small in all cases, and the radiation is dominated by vortex sound at all times. The rate of decay of far-field pressure at late times increases with $\bar{\beta}$, as an outcome of the respective flap motion.

\section{CONCLUDING COMMENTS}

We analyzed the vibroacoustic signature of a twodimensional rigid wing attached to a flap, subject to low-Mach and high-Reynolds (inviscid) number flow, and interacting with a line vortex. A passive-flap configuration was considered, where the flap was attached to the airfoil through a torsion spring, and no external torque was applied to the structure apart from the forcing of the incoming vortex. The coupled fluidstructure interaction problem was analyzed using thin-airfoil theory, and the trailing edge wake was discretized and modeled using the emended Brown and Michael equation. It was found that incident vortex passage above the airfoil excites flap motion at the system natural frequency, which is amplified over all other frequencies contained in the forcing vortex. The consequent far-field acoustic radiation was analyzed using Powell-Howe analogy, yielding a dipole-type leading-order behavior of the system. It was shown that direct flap motion has a negligible effect on the total sound radiation, and that the acoustic field is dominated by the contribution of fluid vorticity at all times. The latter consists of a strong leading and trailing edge interaction of the airfoil with the incident vortex (reduced by the action of trailing edge wake), together with late-time trailing edge vortex-street sound resulting from induced flap motion. The acoustic signature was compared with the counterpart non-flapped (stationary) signal and was found qualitatively different. In particular, it was shown that the flap may amplify or attenuate sound radiation depending on the value of system natural frequency. Sound amplification occurs when system frequency is within the frequency range characterizing vortexairfoil interaction. Meanwhile, a passive mechanism for sound attenuation takes place when flap-fluid natural frequency is outside of this range. This phenomenon was rationalized in terms of Fourier decomposition of the system far-field radiation.

Existing studies on airfoil-flap noise focus mainly on configurations of stationary and slotted flaps, and analyze the effect of slot noise on the airfoil acoustic radiation. In this respect, we consider the present work as complementary to current literature by examining a setup where the flap is fully attached to the airfoil but able to deflect dynamically about its adjoining hinge. As mentioned in Sec. I, related configurations become more relevant nowadays, when novel attached-flap designs are found practically advantageous. It is therefore important to analyze the acoustic properties of such setups.

The present work focuses on a case where the structure is rigid, with only one rotational degree of freedom allowed. This is perhaps the simplest configuration possible to study the effect of non-linear fluid-structure interaction on the system radiation. With increasing need to analyze the vibroacoustic signature of flapping flight, a natural follow-up for this contribution would be the consideration of a more involved model for airfoil elasticity. In addition, the findings of this work may serve as a benchmark model to consider a related noise-control problem, where the system signature will be monitored through external actuation of the flap. This consists of a work currently in progress.

\section{ACKNOWLEDGMENTS}

A.M. acknowledges support by the Marie Curie International Reintegration Grant No. PIRG-GA-2010-276837. L.H. acknowledges the support of a China National Key Basic Research Scheme project No. 2012CB720202.

\section{APPENDIX: SERIES EXPANSION OF $\bar{\gamma}_{\boldsymbol{a}}\left(\overline{\boldsymbol{x}}_{\mathbf{1}}, \overline{\boldsymbol{t}}\right)$}

Using the scaling introduced in Sec. IV, and applying a change of variables $\bar{x}_{1}=-\cos \phi$, the non-dimensional counterpart of the impermeability condition (1) combined with (2) becomes

$$
\frac{1}{\pi} \int_{0}^{\pi} \frac{\bar{\gamma}_{a}(\varphi, \bar{t}) \sin \varphi \mathrm{d} \varphi}{\cos \varphi-\cos \phi}=\bar{F}(\phi, \bar{t}),
$$

where

$$
\begin{aligned}
\bar{F}(\phi, \bar{t})= & 2 H(-\cos \phi-\bar{\eta})\left[-(\cos \phi+\bar{\eta}) \frac{\mathrm{d} \theta}{\mathrm{d} \bar{t}}+\theta\right] \\
& +\operatorname{Re}\left\{\frac{2 \bar{\Gamma}}{\cos \phi+\bar{z}_{\Gamma}}+\sum_{k=1}^{n} \frac{2 \bar{\Gamma}_{k}}{\cos \phi+\bar{z}_{\Gamma_{k}}}\right\}
\end{aligned}
$$

In Eq. (A2), $H(\cdot)$ denotes the unit step function and $\bar{\Gamma}_{k}=\Gamma_{k} /(2 \pi a U)$. Following a standard procedure in thinairfoil theory, we expand $\bar{\gamma}_{a}(\phi, \bar{t})$ using a Fourier-type series

$$
\bar{\gamma}_{a}(\phi, \bar{t})=A_{0}(\bar{t}) \frac{1+\cos \phi}{\sin \phi}+\sum_{m=1}^{\infty} A_{m}(\bar{t}) \sin m \phi,
$$

which satisfies the Kutta condition (13) identically. Substituting Eq. (A3) into Eq. (A1), and making use of the Glauert integral

$$
\frac{1}{\pi} \int_{0}^{\pi} \frac{\cos m \varphi \mathrm{d} \varphi}{\cos \varphi-\cos \phi}=\frac{\sin m \phi}{\sin \phi}
$$

we obtain the equation

$$
A_{0}(\bar{t})-\sum_{m=1}^{\infty} A_{m}(\bar{t}) \cos m \phi=\bar{F}(\phi, \bar{t}) .
$$


The time-dependent coefficients in Eq. (A3) are therefore given by

$$
\begin{aligned}
& A_{0}(\bar{t})=\frac{1}{\pi} \int_{0}^{\pi} \bar{F}(\phi, \bar{t}) \mathrm{d} \phi, \\
& A_{m}(\bar{t})=-\frac{2}{\pi} \int_{0}^{\pi} \bar{F}(\phi, \bar{t}) \cos m \phi \mathrm{d} \phi, \quad m \geq 1 .
\end{aligned}
$$

In practice, our calculations indicate that truncation of the series expansion (A3) at $m=20$ suffices for obtaining a converged result for $\bar{\gamma}_{a}$, with errors $\leqslant 0.1 \%$.

${ }^{1}$ M. J. T. Smith, Aircraft Noise (Cambridge University Press, Cambridge, UK, 1989), $382 \mathrm{pp}$.

${ }^{2}$ M. S. Howe, "On the generation of side-edge flap noise," J. Sound Vib. 80, 555-572 (1982).

${ }^{3} \mathrm{M}$. S. Howe, "On the hydroacoustics of a trailing edge with a detached flap," J. Sound Vib. 239, 801-817 (2001).

${ }^{4}$ Y. P. Guo, "A discrete vortex model for slat noise prediction," AIAA Pap. 2001-2157 (2001).

${ }^{5}$ J. M. Mendoza, T. F. Brooks, and W. M. Humphreys, "An aeroacoustic study of a leading edge slat configuration,” Int. J. Aeroacoust. 1, 241-274 (2002).

${ }^{6}$ Z. C. Zheng, B. K. Tan, and Y. Xu, "Near-field fluctuations and far-field noise of a three-element airfoil system by a discrete vortex method," Appl. Math. Comput. 216, 1072-1086 (2010).

${ }^{7}$ J. W. Delfs, "Reduction technologies and prediction of high lift noise," J. Acoust. Soc. Am. 123, 3393 (2008).

${ }^{8}$ F. Hutcheson, "On the reduction of flap side edge noise," J. Acoust. Soc. Am. 123, 3393 (2008).

${ }^{9}$ T. M. Grundy, G. P. Keefe, and M. V. Lowson, "Effects of acoustic disturbances on low Re aerofoil flows," Prog. Astronaut. Aeronaut. 195, 91-113 (2001).

${ }^{10}$ H. C. Bennet-Clark, "Acoustics of insect song," Nature 234, 255-259 (1971).

${ }^{11}$ J. Sueur, E. J. Tuck, and D. Robert, "Sound radiation around a flying fly," J. Acoust. Soc. Am. 118, 530-538 (2005).

${ }^{12}$ Y. Bae and Y. J. Moon, "Aerodynamic sound generation of flapping wing,” J. Acoust. Soc. Am. 124, 72-81 (2008).

${ }^{13}$ S. Alben, "Optimal flexibility of a flapping appendage in an inviscid fluid," J. Fluid Mech. 614, 355-380 (2008).
${ }^{14}$ S. Michelin and S. G. Llewellyn Smith, "Resonance and propulsion performance of a heaving flexible wing," Phys. Fluids 21, 071902 (2009).

${ }^{15}$ A. Manela and M. S. Howe, "On the stability and sound of an unforced flag," J. Sound Vib. 321, 994-1006 (2009).

${ }^{16}$ A. Manela and M. S. Howe, "The forced motion of a flag," J. Fluid Mech. 635, 439-454 (2009).

${ }^{17}$ A. Manela, "Sound generated by a vortex convected past an elastic sheet," J. Sound Vib. 330, 416-430 (2011).

${ }^{18}$ A. Manela, "Vibration and sound of an elastic wing actuated at its leading edge," J. Sound Vib. 331, 638-650 (2012).

${ }^{19}$ C. L. Streett, J. H. Casper, D. P. Lockard, M. R. Khorrami, R. W. Stoker, R. Elkoby, W. F. Wenneman, and R. James, "Aerodynamic noise reduction for high-lift devices on a swept wing model," AIAA Pap. 2006-212 (2006).

${ }^{20} \mathrm{~F}$. V. Hutcheson, T. F. Brooks, and W. M. Humphreys, "Noise radiation from a continuous moldline link flap configuration," Int. J. Aeroacoust. 10, 565-588 (2011).

${ }^{21}$ I. Wang, S. C. Gibbs, and E. H. Dowell, "Flutter of rectangular plates in three dimensional incompressible flow with various boundary conditions: Theory and experiment," ASME Pap. DETC2012-70952 (2012).

${ }^{22}$ M. S. Howe, Acoustics of Fluid-Structure Interactions (Cambridge University Press, Cambridge, UK, 1998), $571 \mathrm{pp}$.

${ }^{23}$ M. S. Howe, Theory of Vortex Sound (Cambridge University Press, Cambridge, UK, 2003), 216 pp.

${ }^{24}$ C. E. Brown and W. H. Michael, "Effect of leading edge separation on the lift of a delta wing," J. Aeronaut. Sci. 21, 690-706 (1954).

${ }^{25}$ C. E. Brown and W. H. Michael, "On slender delta wings with leadingedge separation," NACA Tech. Note 3430 (1955).

${ }^{26} \mathrm{~N}$. Rott, "Diffraction of a weak shock with vortex generation," J. Fluid Mech. 1, 111-128 (1956).

${ }^{27}$ M. C. A. M. Peters and A. Hirschberg, "Acoustically induced periodic vortex shedding at sharp edged open channel ends: Simple vortex models," J. Sound Vib. 161, 281-299 (1993).

${ }^{28}$ M. S. Howe, "Emendation of the Brown \& Michael equation, with application to sound generation by vortex motion near a half-plane," J. Fluid Mech. 329, 89-101 (1996).

${ }^{29}$ Y. P. Guo, "Application of the Ffowcs Williams/Hawkings equation to two-dimensional problems," J. Fluid Mech. 403, 201-221 (2000).

${ }^{30}$ To study the effect of using the embedded Brown and Michael equation, we have compared our results with those obtained using the original Brown and Michael formula. While some quantitative differences were found, it is worthwhile to note that no qualitative changes were observed. 